\title{
Measurement of Oxygen-Derived Free Radical Generation in the Regionally-Ischemic Rat Heart by the Chemiluminescence Method
}

\author{
Itaru Ohoi, Hiroko Tobari and Keita Nakamura \\ Pharmacology Section, Research Laboratories, Nippon Kayaku Co., Ltd., 31-12, Shimo 3-chome, Kita-ku, Tokyo 115, Japan \\ Received February 18, 1993 Accepted June 17, 1993
}

\begin{abstract}
Generation of oxygen-derived free radicals (oxy-radicals) in the stored rat heart was measured by chemiluminescence. Hearts subjected (ischemia) or not subjected (non-ischemia) to a 4-min regional ischemia were frozen in liquid nitrogen and stored until assayed. The frozen myocardium was ground and oxygenated by mixing it with phosphate-buffered saline $\left(\mathrm{Po}_{2}: 194 \mathrm{mmHg}\right)$ containing lucigenin. The chemiluminescence intensity of ischemic myocardium was larger than that of non-ischemic myocardium. Recombinant human superoxide dismutase significantly decreased these intensities. These results indicate that $\mathrm{O}_{2}{ }^{-}$is one of the major oxy-radical species in the rat heart and that the generation of oxyradicals is enhanced by regional ischemia for $4 \mathrm{~min}$.
\end{abstract}

Keywords: Oxygen radical, Ischemia, Chemiluminescence

We reported that recombinant human superoxide dismutase ( $r-h-S O D)$, a radical scavenger of superoxide anion radical $\left(\mathrm{O}_{2}^{-}\right)$, dose-dependently reduced reperfusioninduced arrhythmia in the anesthetized rat (1). This result indirectly suggested the contribution of oxygen-derived free radicals (oxy-radicals) to the occurrence of reperfusion-induced arrhythmia. However, no direct evidence of oxy-radical generation has been demonstrated in this model. In addition, we developed a simple and reproducible chemiluminescence method for measuring oxy-radicals generated by oxygenation of the stored myocardium, and showed a remarkable increase in oxy-radicals after oxygenation of isolated rat heart subjected to a $30-\mathrm{min}$ global ischemia (2). Therefore, the present study was undertaken to examine oxy-radical generation after oxygenation of the anesthetized rat heart subjected to a 4-min regional ischemia using this chemiluminescence method.

Ten male Sprague-Dawley rats (300 to $450 \mathrm{~g}$ body weight, Charles River Japan, Atsugi) were used. The specific activity of r-h-SOD (Nippon Kayaku, Tokyo) determined according to the method of McCord and Fridovich (3) was $4,120 \mathrm{U} / \mathrm{mg}$ wt. R-h-SOD, albumin (Sigma, St. Louis, MO, U.S.A.), lucigenin ( $N, N^{\prime}$-dimethyl-9,9'-biacridinium dinitrate) (Sigma), hypoxanthine (Sigma) and xanthine oxidase (Boehringer, Mannheim, Germany) were dissolved in phosphate-buffered saline (PBS). The partial pressure of oxygen $\left(\mathrm{Po}_{2}\right)$ in PBS was measured with a blood gas analyzer (Model 288, Ciba-
Corning, Corning, NY, U.S.A.).

Animals were anesthetized with sodium pentobarbital $(55 \mathrm{mg} / \mathrm{kg}$, i.p.), cannulated into the trachea and ventilated with room air by using a respirator ( $\mathrm{SN}-480-7$, Shinano Seisakusho, Tokyo) at a tidal volume of $10 \mathrm{ml} / \mathrm{kg}$ and a ventilatory rate of $60 \mathrm{breaths} / \mathrm{min}$. Atelectasis was prevented by maintaining an expiratory pressure of 2 $\mathrm{cmH}_{2} \mathrm{O}$. The rectal temperature was maintained at $36.5 \pm 0.5^{\circ} \mathrm{C}$ with an infrared lamp. Regional ischemia of the heart was produced according to the previously reported method (1). Briefly, the chest was opened, and the heart was gently exteriorized after pericardiac incision. A ligature (5-0, Kono Seisakusho, Ichikawa) was placed around the left coronary artery at a point close to its origin according to the method of Selye (4), and the heart was repositioned in the thoracic cavity. Both ends of the ligature were passed through a polyethylene tube (ID: 0.4 $\mathrm{mm}$, Length: $50 \mathrm{~mm}$ ) and exteriorized out of the thoracic cavity. After an injection of heparin $(1000 \mathrm{U} / \mathrm{kg}$, i.v.), both ends of the ligature were pulled, and consequently, one side of the polyethylene tube facing to the heart was made to press against the left coronary artery. The tube and ligature were clamped together with a small hemostat for $4 \mathrm{~min}$, because the protective effect of r-h-SOD on reperfusion-induced arrhythmia was observed in rats subjected to the same 4-min regional ischemia (1). After the 4-min ischemia, a lump of the paled myocardium was cut off with a pair of scissors and immediately immersed in liq- 
uid nitrogen (ischemia). Sham operations were performed by the same procedure except for the ligation of the left coronary artery (non-ischemia). The frozen hearts were stored at $-80^{\circ} \mathrm{C}$ for about 1 week until assayed. According to our preliminary examination on the influence of the preservation period on chemiluminescence intensities of oxygenated ischemic myocardium, no difference was observed between myocardium prepared freshly and that preserved at $-80^{\circ} \mathrm{C}$ for 1 month.

Non-ischemia and ischemia groups consisted of five frozen hearts each, and each heart was divided into three different treatment groups (control, albumin, and I-hSOD). These hearts were oxygenated according to the previously reported method (2). Briefly, each frozen heart was put in a teflon capsule containing a tungsten-carbide ball, which had been previously immersed in a liquid nitrogen bath, and ground for $30 \mathrm{sec}$ with a microdismembrator (B. Braun, Melsungen, Germany). About $40 \mathrm{mg}$ of the frozen and ground tissue was weighed exactly ( 37 to $43 \mathrm{mg}$ ) and transferred to a test tube. One milliliter of PBS (pH 7.4, $\mathrm{Po}_{2}: 194 \mathrm{mmHg}, 37^{\circ} \mathrm{C}$ ) containing $1 \mathrm{mg}$ of lucigenin (control) and, additionally, $0.5 \mathrm{mg} / \mathrm{ml}$ albumin or $2,000 \mathrm{U} / \mathrm{ml} \mathrm{r}$-h-SOD was added to the tube, and the mixture was vortexed immediately for $10 \mathrm{sec}$ to thaw and oxygenate the myocardium. Albumin was used to examine the influence of $r-h-S O D$ as protein on chemiluminescence intensity. Two thousand units of r-h-SOD contains the same amount of protein as $0.5 \mathrm{mg}$ of albumin. The mixed solution was injected into a chemiluminescence detection flow cell (CLD-100, Tohoku Electronic
Industrial Co., Ltd., Sendai) $15 \mathrm{sec}$ after vortexing. Chemiluminescence was recorded on a desktop recorder (RC-250, Jasco, Tokyo) for $5 \mathrm{~min}$. The area under each 5-min tracing was measured with a digitizer (KD4300, Graphtec, Tokyo) to quantify the chemiluminescence intensity. The area under a 5 min-tracing obtained from a mixed solution of hypoxanthine $(10 \mu \mathrm{M})$, xanthine oxidase $(10 \mathrm{mU} / \mathrm{ml})$ and lucigenin $(1 \mathrm{mg} / \mathrm{ml})$ at $37^{\circ} \mathrm{C}$ was defined as one chemiluminescence unit (CU) in this study. The chemiluminescence intensity obtained from each solution was expressed as $\mathrm{CU} / \mathrm{g}$ wet weight tissue.

All data are expressed as the mean \pm S.E.M. Student's $t$-test was carried out to determine any differences between the non-ischemic and ischemic groups. A one way analysis of variance (ANOVA) was carried out to determine any differences among the control, albumin-treated, and r-h-SOD-treated groups. When a statistically significant difference was observed, individual means were compared by Tukey's multiple comparison test. A probability of $<5 \%$ was considered significant.

Increases in chemiluminescence were observed after injections of PBS containing lucigenin and either nonischemic or ischemic myocardium into the cell (Fig. 1). These chemiluminescences reached maximal levels immediately after the injections and then decreased exponentially. The chemiluminescence intensity of oxygenated ischemic myocardium was significantly larger $(P<0.001)$ than that of oxygenated non-ischemic myocardium (Fig. 2). R-h-SOD significantly reduced the chemiluminescence intensities of oxygenated ischemic myocardium $(P<0.01)$
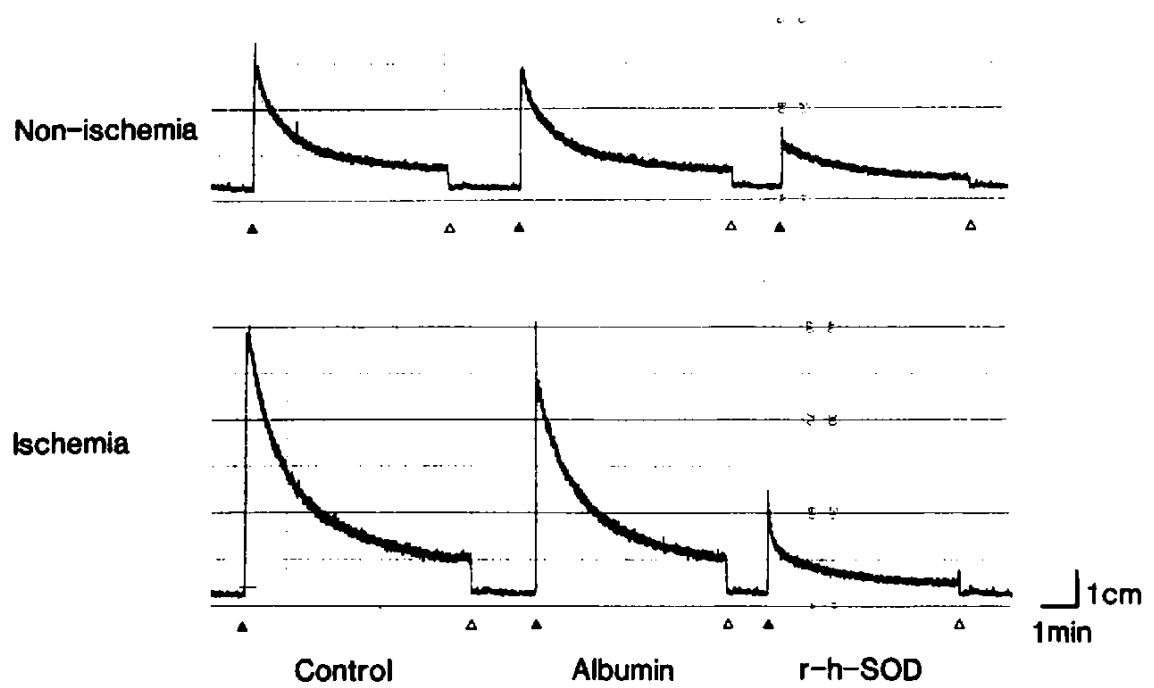

Fig. 1. Actual tracings of chemiluminescence response to oxygenated myocardium. To thaw and oxygenate frozen and ground myocardium, the myocardium (about $40 \mathrm{mg}$ ) was mixed with $1 \mathrm{ml}$ of PBS containing $1 \mathrm{mg} / \mathrm{ml} \mathrm{lucigenin} \mathrm{(control)} \mathrm{and} 0.5$ $\mathrm{mg} / \mathrm{ml}$ albumin or $2,000 \mathrm{U} / \mathrm{ml} \mathrm{r}-\mathrm{h}-\mathrm{SOD}$. The mixed solution was injected into a chemiluminescence detection flow cell and the chemiluminescence was recorded. Closed and open triangles show the injections of the mixed solution and water, respectively. 


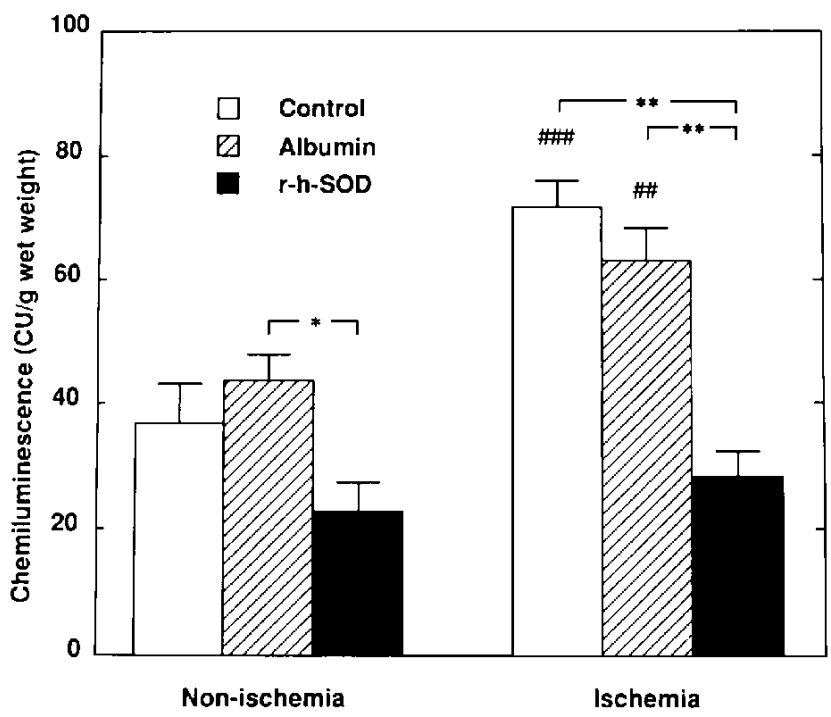

Fig. 2. Chemiluminescence intensity of oxygenated non-ischemic and ischemic myocardium and the effects of albumin and r-h-SOD. Values are the means $\pm S$.E.M. of 5 hearts. $\mathrm{CU}$; chemiluminescence unit described in the text. ${ }^{*} \mathrm{P}<0.05$ and ${ }^{* *} \mathrm{P}<0.01$, compared with the control, albumin-treated, and r-h-SOD-treated groups by Tukey's multiple comparison test after ANOVA. ${ }^{* \prime} \mathrm{P}<0.01$ and ${ }_{\pi \rightarrow 4}{ }^{74} \mathrm{P}<0.001$, compared with non-ischemia by Student's $t$-test.

and oxygenated non-ischemic myocardium, but albumin showed no influence in either myocardium (Fig. 2).

In this study, rat hearts which were subjected to (ischemia) or not subjected (non-ischemia) to regional ischemia for $4 \mathrm{~min}$ were frozen in liquid nitrogen and stored at $-80^{\circ} \mathrm{C}$ for 1 week until assayed. Oxy-radicals generated during the experiments could not be measured by the present method because of their instability even at $-80^{\circ} \mathrm{C}$ (5). Therefore, the purpose for freezing the myocardium was not to prolong the life of the generated oxy-radicals, but to preserve the myocardial ability to generate oxy-radicals. The stored frozen hearts were ground and then oxygenated by mixing with PBS containing lucigenin. The oxygen tension in intact myocardium is reported to be 15 to $25 \mathrm{mmHg}(6)$. Therefore, PBS in which oxygen was maintained at $194 \mathrm{mmHg}$ contains enough oxygen to oxygenate myocardial tissue.

The chemiluminescence intensity of oxygenated ischemic myocardium was significantly larger than that of oxygenated non-ischemic myocardium. This indicates that the myocardial ability to generate oxy-radicals was significantly increased by a 4-min regional ischemia of the heart. $R$ $h$-SOD reduced the chemiluminescence intensities of oxygenated non-ischemic and ischemic myocardium. Furthermore, lucigenin, which was used as a chemiluminescence enhancer, is known to be very sensitive to $\mathrm{O}_{2}{ }^{-}$(7). Therefore, $\mathrm{O}_{2}^{-}$seemed to be a predominant radical in this model. However, a fair amount of chemiluminescence was still observed despite the application of r-h-SOD at a high concentration of $2,000 \mathrm{U} / \mathrm{ml}(1,2)$. This suggests the presence of non-specific luminescence which is not degraded by SOD $(1,8)$.

Several possible sources of $\mathrm{O}_{2}^{-}$production in oxygenated ischemic hearts have been proposed, including xanthine oxidase (9), leakage from mitochondrial electron transport chains (10), oxidation of catecholamines (11), and so on. Furthermore, in the present in vivo model, leukocytes in the blood also seem to be another major source of $\mathrm{O}_{2}{ }^{-}$production (12). However, it is unclear which mechanism actually contributes to $\mathrm{O}_{2}{ }^{-}$production in this model, and which mechanism could be preserved at

$80^{\circ} \mathrm{C}$ for 1 week. Further work is needed to identify both these mechanisms.

In this study, we have shown that $\mathrm{O}_{2}{ }^{-}$is generated by oxygenation of frozen rat heart and scavenged by r-hSOD. Furthermore, in a previous study, we showed that $r$-h-SOD dose-dependently reduced the incidence of reperfusion-induced irreversible arrhythmia using the same rat model (1). Therefore, it can be concluded that $\mathrm{O}_{2}{ }^{-}$plays an important role in the occurrence of reperfusion-induced arrhythmia in this model.

\section{REFERENCES}

1 Ohoi, I., Ozawa, N. and Nakamura, K.: Effect of recombinant human superoxide dismutase ( $\mathrm{r}-\mathrm{h}-\mathrm{SOD}$ ) on reperfusion-induced irreversible arrhythmia in anesthetized rats. Folia Pharmacol. Japon. 101, 93-99 (1993) (Abs. in English)

2 Ohoi, I., Sone, K., Tobari, H., Kawano, E. and Nakamura, K.: A simple chemiluminescence method for measuring oxygenderived free radicals generated in oxygenated rat myocardium. Japan. J. Pharmacol. 61, 101-107 (1993)

3 McCord, J.M. and Fridovich, I.: Superoxide dismutase: an enzymic function for erythrocuprein (hemocuprein). J. Biol. Chem. 244, 6049-6055 (1969)

4 Selye, H., Bajusz, E., Grasso, S. and Mendell, P.: Simple techniques for the surgical occlusion of coronary vessels in the rat. Angiology 11, 398-407 (1960)

5 Zweier, J.L., Flaherty, J.T. and Weisfeldt, M.L.: Direct measurement of free radical generation following reperfusion of ischemic myocardium. Proc. Natl. Acad. Sci. U.S.A. 84, 1404- 1407 (1987)

6 Winbury, M.M., Howe, B.B. and Weiss, H.R.: Effect of nitroglycerin and dipyridamole on epicardial and endocardial oxygen tension - further evidence for redistribution of myocardial blood flow. J. Pharmacol. Exp. Ther. 176, 184-199 (1971)

7 Murphy, M.E. and Sies, H.: Visible-range low-level chemiluminescence in biological systems. Methods Enzymol. 186, $595-610(1990)$

8 Takahashi, A., Nakano, M., Mashiko, S. and Inaba, H.: The first observation of $\mathrm{O}_{2}^{-}$generation in in situ lungs of rats treated with drugs to induce experimental acute respiratory distress syndrome. FEBS Lett. 261, 369-372 (1990)

9 McCord, J.M.: Oxygen-derived free radicals in postischemic 
tissue injury. N. Engl. J. Med. 312, 159-163 (1985)

10 Boveris, A. and Cadenas, E.: Mitochondrial production of superoxide anions and its relationship to the antimycin insensitive respiration. FEBS Lett. 54, 311-314 (1975)

11 Singal, P.K., Kapur, N., Dhillon, K.S., Beamish, R.E. and Dhalla, N.S.: Role of free radicals in catecholamine-induced cardiomyopathy. Can. J. Physiol. Pharmacol. 60, 1390-1397 (1982)

12 Fantone, J.C. and Ward, P.A.: Role of oxygen-derived free radicals and metabolites in leukocyte-dependent inflammatory reactions. Am. J. Pathol. 107, 397-418 (1982) 